\title{
APUNTES SOBRE LA CONFIGURACIÓN ACTUAL DEL ETHOS MÉDICO
}

\begin{abstract}
Luis Risco*
Resumen: El perfil que en términos éticos ha ido adquiriendo el rol del médico en la sociedad actual no ha sido considerado suficientemente como objeto de análisis ni entre el mismo cuerpo médico ni por otras instancias de reflexión, aun cuando los efectos de este insensible pero significativo cambio se dejan ver en múltiples aspectos del ejercicio de la profesión médica. Por de pronto, en la "legalización" de la actividad médica, en el cambio de la relación médico-paciente a otra del tipo médicocliente, en la presión que ejerce la medicina administrada sobre la temporalidad del acto médico, etc., situaciones en las cuales se pueden notar de manera palmaria manifestaciones de lo señalado. Este artículo pretende presentar ciertas bases para una reflexión sobre el tema, desde la perspectiva del "estar en el mundo" que eventualmente correspondería al rol del médico.
\end{abstract}

Palabras clave: ética médica, humanismo médico

\section{SKETCHES ABOUT NOWADAYS' MEDICAL ETHOS}

\begin{abstract}
In ethical terms the profile that the doctor's rôle has acquired in today's society has not been sufficiently considered as an analysis' object neither among the medical corps nor in other reflection instances, even though the effects of this insensible but significant change can be noticed in multiple aspects of the medical professional practice. To begin with, in the "legalization" of the medical activity, in the path from a doctor-patient relationship to another one of the type doctor-client, in the pressure that the administrated medicine puts on the temporality of the medical act, etc., situations through which you can clearly see manifestations of what has just been pointed out. This paper pretends to set certain basis that would lead to consider the subject from the "to be in the world" perspective that, eventually, corresponds to the doctor's rôle.
\end{abstract}

Key words: medical ethics, medical humanism

\section{APONTAMENTOS SOBRE A CONFIGURAÇÃO ATUAL DO ETHOS MÉDICO}

Resumo: O perfil ético que vem adquirindo o papel do médico na sociedade atual não tem sido considerado suficientemente objeto de análise, sequer pelo próprio corpo médico, além de outras instâncias de reflexão, ainda quando os efeitos desta insensível, porém significativa mudança, tornam-se visíveis nos múltiplos aspectos do exercício da profissão médica. Uma, de imediato, na "legalização" da atividade médica, na mudança da relação médico-paciente; a outra, do tipo médico-cliente, na pressão que exerce a medicina administrada sobre a temporalidade do ato médico etc., situaçôes nas quais se pode notar, de modo palmar, manifestaçôes do que foi assinalado. Este artigo pretende apresentar certas bases para uma reflexão sobre o tema, a partir da perspectiva do "estar no mundo" que eventualmente corresponde ao papel do médico.

Palavras-chave: ética médica, humanismo médico

Médico Psiquiatra. Profesor de Psiquiatría de la Facultad de Medicina, Universidad de Chile. Subdirector de la Clínica Psiquiátrica Universitaria. Chile Correspondencia: 1risco@redclinicauchile.cl 


\section{Ethos}

El ámbito de la ética está delimitado, en términos generales, por la reflexión atingente a aquello que denominamos moral y que se refiere, en última instancia, a cuál es la posición más ecuánime, mirando desde todos los puntos de vista posibles, que la comunidad debiera tomar con relación a algún aspecto sensible, con diferentes valencias derivadas de los distintos enfoques axiológicos que pudieran estar poniéndose en juego en una determinada circunstancia.

Lo señalado define los límites. Pero si se tratase de determinar la esencia de la reflexión ética, cabría aceptar necesariamente que está dirigida sobre lo que los griegos denominaron ethos: "el lugar desde el que manan nuestras propias facultades". Es posible describir el ethos como una noción que refleja una "posición" o "actitud" con respecto al mundo y a sí mismo, una cierta manera de "estar en el mundo", desde la cual surgen naturalmente ciertos atributos y características. Es posible suponer que el ejercicio ético de una disciplina o profesión implica no sólo las destrezas y competencias técnicas atingentes, sino también una manera de disponerse sobre la realidad, una forma de mirarla que, como consecuencia directa, influencia esas competencias y las regula en relación con los demás y con la naturaleza toda. En estos términos, el ethos viene a ser algo así como el fundamento ontológico de una profesión y el elemento esencial que dictamina cómo ha de disponerse la persona para ejercer esa profesión, y cómo las personas que ejercen esa profesión deben comportarse con el fin de alcanzar la plenitud de la expresión del arte o ciencia que profesan.

La profesión militar, por ejemplo, o la de las antiguas órdenes guerreras exige y exigió la marcialidad como parte fundamental de la actitud para la dedicación verdadera a esa profesión. Hay cientos de referencias a este aspecto en la historia, siendo quizás "El libro de los Cinco Anillos" de Miyamoto Musashi(1) una de las más conspicuas. La marcialidad podría entenderse como una actitud de estoicismo y acatamiento del deber por encima de todas las cosas, incluso la vida y la familia. Esta actitud ha alcanzado el rango de "camino" en algunos casos, cuando a los valores de la marcialidad se han añadido los elementos del misticismo. La marcialidad como actitud supone una manera de enfrentar la realidad y prescribe los códigos de conducta cotidianos; en situaciones límite, define con sencillez qué cabe y qué no cabe en la vida de un hombre marcial, y señala una dirección sobre la que hacer la vida. Pareciera que, en algunos casos, el ethos alcanzara un valor superior al de la persona, como si tuviese una especie de vida propia, y que, en la medida en que está mejor configurado, un hombre que dedica su vida a él encontrará un verdadero sentido, con o sin una creencia acerca de la existencia o inexistencia de alguna divinidad.

\section{El sacerdocio}

En todas las épocas y lugares han surgido en las sociedades humanas figuras que presentan poca variación de lugar en lugar y alrededor de muchas de las cuales se estructura la vida social. Una de ellas es la del "sacerdote", mediador entre las fuerzas "más allá de este mundo" -sean cuales sean- y la vida cotidiana de los seres humanos. La figura del sacerdote se entremezcla en ocasiones con la del chamán o brujo, otra figura que representa a un hombre capaz de ir al reino de los dioses, conocer sus acontecimientos y traer nuevas desde él a este mundo. Ser chamán es, por tanto, una tarea delicada, pues existe el riesgo de perder el camino, y se requiere una enorme disciplina interior y el conocimiento de ciertas técnicas de origen esotérico. En ciertos casos, la figura del chamán se difumina a veces con la del bardo, al cual se reconoce en principio tan solo la propiedad de "ver" el mundo de la mitología, pero que en algunas tradiciones se enriquece con una capacidad de ir y volver desde el mundo de los dioses. Arturo, el rey de los bretones, caído en la batalla de Calmann, es guiado a su destino final, la isla de Avalon, por dos bardos, quienes conocen ese misterioso camino(2).

En ambos casos, el del sacerdote y el chamán, la dedicación a la disciplina que supone cada una de estas figuras no descansa solamente en la adquisición de un bagaje de destrezas, habilidades y conocimientos. También se requiere de una actitud y un modo de "estar en el mundo", desde el cual surgen las facultades de cada cual, y que impone una determinada estructuración y ciertas limitaciones al modo de existir. Por ejemplo, es imposible pensar en un sacerdote o chamán juerguistas o de costumbres lujuriosas; su poder entre los hombres y en relación con el mundo al cual remiten se reduciría considerablemente.

Al sacerdote se le exige capacidad de posponerse, tiempo para la meditación y un sentido acendrado sobre lo que corresponde y lo que no en cualquier situación, y 
todo esto no sólo en lo que respecta al ejercicio del rol sacerdotal sino en la vida entera. Quizás podría decirse que hay otras disciplinas para las cuales sólo bastaría con las habilidades necesarias para su completo ejercicio, sin que se requiera de una determinada actitud esencial. Quizás haya otras para las cuales se requiere de una cierta manera de ser sólo en tanto el rol está en ejercicio, como por ejemplo en el caso de un asesor. Pero al sacerdote se le exige que haga la vida entera, hasta en sus más mínimos detalles, con arreglo a su rol. A su vez, y probablemente en relación estrecha con este grado de exigencia, al sacerdote se le entregan ciertas credibilidades muy por encima de las que se atribuiría a cualquier otra figura o rol. Nadie discutiría una prescripción sacerdotal, nadie la pondría en entredicho, pues ello está enteramente fuera de lugar.

Esta "manera" y estas atribuciones están en concordancia con el papel del sacerdote en la comunidad, que es el de cautelar el sentido de lo sacro, de suyo emergente en toda sociedad. Hasta ahora no se conocen sociedades en las cuales no aparezca con fuerza a veces sorprendente el sentido de lo sacro. Y aun cuando en el Occidente actual este sentido se encuentre de cierta manera en una especie de crisis -Kakar, psicoanalista hindú, reflexiona acerca de "un duelo occidental por la pérdida de lo sagrado" (3)-, se sigue exigiendo y reconociendo, con más o menos matices, las facultades y limitaciones propias del rol del sacerdote y que derivan directamente de aquello que corresponde a su ethos.

El cuidado exitoso que un sacerdote haga de sí mismo en cuanto tal posibilita una serie de formas de relación que son únicas y características de su función. La confesión, por ejemplo, que tiene lugar frente al sacerdote, se distingue de varias maneras de otros tipos de actos semejantes. Tiene el carácter del reconocimiento pleno que antecede a la expiación, una secuencia que posibilita el perdón del sí mismo, más aún si tiene lugar en el ambiente sacro, frente a una instancia superior. Este acto, quizás el de más profunda intimidad frente a un otro, sólo puede tener lugar con alguien que tiene la calidad de sacerdote, que tiene esa manera de comportarse en cada acto vital y que, por lo tanto, está "parado en un lugar" desde el cual es natural que pueda ser "guardián" de lo sacro y de aquello que se ha aludido como "alma", "espíritu", "fondo anímico", en fin, aquello que entronca con las raíces inconscientes de la actividad psíquica y que $\boldsymbol{s} \boldsymbol{e} \boldsymbol{h} \boldsymbol{a}$ querido denominar como "arquetípico".
Muchos han sido los estilos de sacerdocio a través de la historia y de los tiempos. Las figuras de las vestales intoxicadas por vapores sulfurosos en Delfos poco se parecen a las de los misioneros que se internaban en las selvas del Amazonas. Sin embargo, su relación con quienes necesitan de las actitudes y las facultades de los sacerdotes no parece presentar grandes variaciones con el paso de las épocas. La voz de los sacerdotes, más intensa cuanto más su vida se acerca a la pureza del correspondiente ethos, es oída sin recelo, con la calidad de una profecía, suscitando el recogimiento y la entrega. Tan solo la voz del sacerdote es portadora de estos atributos, y ninguna, en lugar alguno, se le llega a parecer. La voz del bardo se oye como si viniese de un sueño; la del chamán como si trajese datos de un mundo "más alla", que fundamenta este mundo cotidiano pero que tiene poco que ver con él; la voz del sacerdote es omnipresente, configuradora e incluso se le podría reconocer una suerte de carácter fundacional. En toda actividad que se espera sea para siempre es necesaria la palabra de un sacerdote, como si esa palabra contuviese algo de eternidad.

\section{El médico}

Nos hemos detenido con cierto detalle en la figura del sacerdote porque tradicionalmente el ethos del médico ha limitado siempre con el de aquél y muchos le reconocen estar imbuido de varias de sus atribuciones y de más de algo del temple esencial. La figura del médico, más allá de que las prácticas y la orientación de las destrezas y habilidades sean fundamentalmente distintas de las del sacerdote, se parece en varios aspectos que no son irrelevantes. El sacerdote tiene una acabada noción del tramo entre la vida y la muerte, un tramo sobre el cual el quehacer cotidiano del médico está precisamente enfocado, para intervenir sobre él de la mejor manera posible. La confesión ante el sacerdote tiene el sentido de "sanar" el espíritu, dejarlo libre de una cierta forma de esclavitud -la de la culpa-, mientras que la confesión ante el médico también ha de liberar de una cierta forma de esclavitud, la que sobreviene con la enfermedad. En ciertas áreas de la medicina esta similitud es aún más acentuada, como en la psiquiatría. Muchos psiquiatras con años de experiencia han percibido una y otra vez cómo la simple expresión de una culpa, una fantasía destructiva, un sentimiento innoble, bastan para el alivio y para dar comienzo a una nueva manera de experimentar la propia realidad. Es por aspectos como los reseñados que las figuras del 
médico y el sacerdote tienen una reconocida imbricación y, podría decirse, una señalada semejanza en ciertas áreas de un ethos común.

Sin embargo, la figura y el papel que la sociedad atribuye hoy al médico han cambiado. La palabra del médico ya no tiene aquella tradicional similitud con la del sacerdote en muchos sentidos, y esto ha reformulado de manera quizás irreversible la estructura de la relación médico-paciente y también el ethos del médico actual. Las consecuencias no han sido suficientemente estudiadas y explicitadas, y es probable que incluso dentro del mismo cuerpo médico no haya plena conciencia de cuán diferente es la manera como distintas generaciones de médicos experimentan la medicina en cuanto manera de existir. Por de pronto, la relación contractual entre el médico y el paciente hoy está intervenida por un tercero, los dueños de la medicina administrada, que actúan como moduladores de esa relación sin tener el mismo interés que el médico y el paciente en el resultado exitoso en términos de salud. Y aún más, hay terceros y cuartos que tienen interés en esta relación, pero a efectos de sacar explícito provecho pecuniario de ella, como son los que incitan a los pacientes a demandar a los médicos bajo cualquier pretexto y subterfugio. Todos estos hechos son indicadores definitivos de que el médico y el ejercicio de su profesión hace ya tiempo fueron expulsados irremisiblemente de la casa del sacerdote. Es posible que el ethos del médico haya migrado desde el carácter sacerdotal que lo investía hacia otro que se parece en mucho al de un "asesor", alguien que aconseja, que sopla al oído qué es lo mejor que se podría hacer en una determinada situación, y que luego acompaña en una decisión que el paciente mismo y no él ha tomado.

¿Podría decirse que hay un ethos del asesor que alcanza la misma profundidad e intensidad en la determinación de una manera de existir que la del sacerdote o la del médico tradicional? Es difícil sostenerlo. La calidad de asesor supone varias limitaciones centradas en la idoneidad, la honestidad y el conflicto de intereses, pero no se puede afirmar que la calidad de asesor obligue a vivir de una cierta manera sin la cual el ejercicio del rol se debilitaría. Mientras el sacerdote cuida de lo esencial del ser más allá de los atributos y riquezas, el asesor en general suele cuidar de tales atributos y riquezas más que de lo esencial de una persona. Su relación es con un cliente, alguien que lo contrata para alcanzar determinados fines. El sacerdote se relaciona con un creyente, alguien que le pide guía para purificar y honrar su espíritu; el médico con un paciente, alguien doblegado por la enfermedad, que se entrega y que por diversas razones no está en situación de decidir por sí mismo ante la tal enfermedad. El cambio de los tiempos ha provocado que-sin que los médicos hayan podido hacer demasiado para detener esa tendencialos pacientes hayan ido insensiblemente adquiriendo la calidad de clientes. Y por tanto, en la hora final, han quedado solos frente a la enfermedad.

Hay por cierto notables diferencias entre la relación médico-cliente y la relación médico-paciente, muchas de las cuales son de natural obviedad. Por ejemplo, en lo que se refiere a la asistencia espiritual frente a un momento de amenaza a la vida o frente a una crisis biográfica mayor, alguien de quien se es cliente podrá sugerir y predecir con mayor o menor juicio y precisión, pero en el límite de la experiencia determinada por el acontecimiento provocador $-\mathrm{y}$ aún más allá- el individuo se encuentra a solas, sin otra compañía que él mismo. Lo contrario ocurre cuando se es paciente, es decir, cuando se ha hecho entrega de las decisiones límite a otro, el cual, además de estar investido de las habilidades y destrezas referidas a la salud y la enfermedad, asume la vocería y toma las decisiones frente a la extrema dicotomía de la vida y la muerte. En la calidad de paciente se puede reconocer una entrega plena, semejante a la que corresponde frente a un sacerdote, basada en una relación de profunda e íntegra confianza y reconocimiento, distinta a la que se establece cuando sencillamente se entrega o contrata un servicio determinado.

\section{El cambio}

Sin duda, el cambio que ha venido ocurriendo en la estructura de la relación médico-paciente no sólo ha tenido consecuencias operacionales. La posibilidad de interpelación de los actos médicos ha hecho surgir la necesidad del médico de "ponerse a cubierto", de tomar medidas en el acto médico que no sólo vayan en beneficio del paciente, sino también destinadas a precaver una evaluación crítica de esas medidas, tomadas en muchos casos en situaciones de emergencia, en las cuales la rapidez de una decisión exige tomar el camino del mal menor. Asimismo, el médico debe considerar que los eventos fortuitos o impredecibles puedan ser interpretados como negligencia, con las consecuencias legales que ello podría suponer. Circunstancias como 
estas redundan necesariamente en que el médico no se sienta en propiedad para tomar decisiones riesgosas que podrían, en un momento dado, salvar una vida, y que en ese caso pueda ser preferible evitar los riesgos personales, aun frente a la posibilidad de complicar el pronóstico del paciente.

Evidentemente, este estado de cosas tiene algunas ventajas. Por de pronto, limitar el ejercicio de quienes pudieran no estar habilitados de manera conveniente para aplicar una técnica o diseñar un plan terapéutico específico. Pero, por otra parte, deja en una especie de abandono a la persona que solicita ayuda. Eventualmente, dejan de ser parte del acto médico propiamente tal el consuelo, la condolencia, la compasión, aspectos que han sido esenciales de la profesión médica por siglos y que en ciertas circunstancias son más valiosos que la habilidad técnica en sí, como por ejemplo en los casos de pacientes terminales o en aquellos en los cuales no queda más que aliviar. El cliente, en la situación más extrema, quedaría solo ante sí, con el apoyo nada más que de alguien que le provee de soporte técnico y a quien no le corresponde hacer más que lo que está prescrito que haga esencialmente en esos términos. Con la pérdida del carácter sacerdotal de la profesión, con el cambio del ethos del médico en la época actual, definitivamente no sólo ha perdido el médico.

Es evidente que una generalización como la propuesta tiene una serie de limitaciones. Por ejemplo, es imposible que el acto médico deje radicalmente de ser una relación personal, en la cual emergen de manera natural sentimientos nobles de la más diversa índole, más aún cuando uno de los participantes se ha preparado para llevar una existencia entera centrada en ayudar a los demás. Pero, por otra parte, es posible que la variación de la estructura relacional entre médico y paciente en la época actual -y las consecuencias que ello tiene en lo que hemos denominado ethos del médico- hayan sido subvaloradas, y tal vez sea necesaria una visión como ésta para poder contrastar mejor el fenómeno. El cambio de esta estructura destacada aquí con alguna exageración -por lo demás, la necesaria para enfatizar convenientemente un cierto ángulo de visión- tiene una miríada de otras consecuencias en las más diversas direcciones. La intervención de aspectos legales, la relación entendida como una transacción de servicios con las regulaciones sociales que eso supone, entre otras, son algunas de estas consecuencias. Pero quizás la más esencial esté relacionada con la posible desaparición de los aspectos sacerdotales de esta relación, lo que deja en una especie de indefensión a pacientes que, en un momento crucial, los necesitan de manera extrema.

\section{A modo de conclusiones}

La relación médico-paciente ha ido adquiriendo insensiblemente, y por momentos de manera ostensible, los caracteres de desacralización con los que el hombre occidental ha construido su mundo. Por esa vía y por efecto de la intervención de agentes que no son propiamente participantes directos de esa relación, el ethos del médico actual ha ido perdiendo los atributos sacerdotales que en alguna época tuvo y que le fueron reconocidos desde antańo. Con ello se extravía uno de los que fueran fundamentos esenciales de la ética del médico, cual es el carácter sacro de la profesión. Este carácter, junto al del arte clínico, ha sido desde siempre uno de los fundamentos humanistas del ethos tradicional del médico.

El temple actual del ethos del médico, en este contexto, aún está en vías de configuración: un fenómeno cuya amplitud de consecuencias todavía no se considera en toda su extensión. Por ejemplo, el trato de la medicina administrada hacia el médico es el que se da a un prestador, una forma de asesoría referida a la entrega de determinados servicios. Esto supone -en una variedad de contextos- una exigencia de rendimientos administrativos que muchas veces choca con los de la buena práctica médica, dictados desde el ethos tradicional del médico.

El propósito de este artículo es proponer ciertas bases para una reflexión en este ámbito, una reflexión que seguramente ha de tener señaladas consecuencias sobre el tipo de existencia que corresponde llevar a los médicos hoy y también sobre nuevas posibilidades que aparecen y sobre otras que desaparecen. Una reflexión final y definitiva debiera incorporar necesariamente elementos de diversas fuentes, incluyendo la del futuro de la profesión, que en definitiva remite, en una dimensión más personal, a la manera de existir que habrán de llevar aquellos que escojan la medicina como su esencial propósito vital. Quizás el médico actual deba asumir que le corresponde abandonar la casa del sacerdote, con las oportunidades y limitaciones que ello supone. Pero antes deberá forzarse necesariamente en una reflexión que excede con mucho la mirada con la que hoy asiste. 
Apuntes sobre la configuración actual del ethos médico - Luis Risco

\section{Referencias}

1. Musashi M. El libro de los cinco anillos. México: Lectorum; 2006.

2. De la Villemarqué Hersart. El Misterio Celta. España: José de Olañeta; 1999: 47.

3. Kakar Sudhir. Chamanes, misticos y doctores. México: Fondo de Cultura Económica; 1989: 15.

Recibido: 6 de octubre de 2008

Aceptado: 28 de octubre de 2008 